\title{
Semi-gas kinetics model for performance modeling of flowing chemical oxygen-iodine lasers (COIL)
}

\author{
GAO Zhi, HU Limin \& SHEN Yiqing \\ Institute of Mechanics, Chinese Academy of Sciences, Beijing 100080, China \\ Correspondence should be addressed to Gao Zhi (email: gaozhi@imech.ac.cn) \\ Received November 21, 200
}

\begin{abstract}
A semi-gas kinetics (SGK) model for performance analyses of flowing chemical oxygen-iodine laser (COIL) is presented. In this model, the oxygen-iodine reaction gas flow is treated as a continuous medium, and the effect of thermal motions of particles of different laser energy levels on the performances of the COIL is included and the velocity distribution function equations are solved by using the double-parameter perturbational method. For a premixed flow, effects of different chemical reaction systems, different gain saturation models and temperature, pressure, yield of excited oxygen, iodine concentration and frequency-shift on the performances of the COIL are computed, and the calculated output power agrees well with the experimental data. The results indicate that the power extraction of the SGK model considering 21 reactions is close to those when only the reversible pumping reaction is considered, while different gain saturation models and adjustable parameters greatly affect the output power, the optimal threshold gain range, and the length of power extraction.
\end{abstract}

Keywords: chemical oxygen-iodine laser (COIL), semi-gas kinetics (SGK) model, rate equation (RE) model, homogeneous broadening, inhomogeneous broadening, performance analysis, spectral line broadening (SLB) model.

DOI: 10.1360/03yw0165

The gas pressure in a laser cavity of flowing chemical oxygen-iodine laser (COIL) is about 133.3-1333 $\mathrm{Pa}^{[1]}$. In this pressure range, effects of homogeneous and inhomogeneous broadening competing with each other on the performances of a COIL are noticeable. Generally, a rate equation (RE) model is adopted to the performance modeling of a supersonic COIL, such as in refs. [1-4]. This model assumes the spectral line profile is homogeneously broadened. Homogeneous broadening assumption simplified greatly the treatment of problems, and the reasons of adopting homogeneous broadening model to the condition of inhomogeneous broadening being predominant in the HF and DF chemical laser were discussed in ref. [5]. 
However, the RE model cannot consider frequency characteristics of the spectral line profile and cannot explain the anomalous gain saturation in HF/DF chemical laser experiments ${ }^{[5,6]}$. A semi-gas kinetics (SGK) model ${ }^{[6-8]}$ can solve simultaneously the continuous medium equations for the macroscopic gas flow and the velocity distribution function equations for the lasing energy level particles. Thus, the SGK model could predict exactly the anomalous gain saturation and anomalous saturation line profile in HF chemical lasers ${ }^{[9,10]}$, and the gain saturation theory of gas lasers (there is no macroscopic flow in it) and the RE model of gas flow lasers were included in it as special cases. The performance calculation of supersonic HF/DF chemical lasers indicated that there is obvious difference between the performance predictions of the SGK model and the RE model when both homogeneous broadening and inhomogeneous broadening are of importance, especially when inhomogeneous broadening is predominant ${ }^{[6-8]}$.

The gas pressure range in a supersonic COIL cavity is nearly the same as that in the HF/DF chemical lasers ${ }^{[1,5]}$. Therefore, in order to optimize the adjustable parameters of a supersonic COIL, it is necessary to deduce the SGK model for performance analyses of a supersonic COIL and compare performances predicted by the SGK model with those by the RE model. Apparently, in the development of supersonic HF/DF chemical lasers and supersonic COIL it is quite important to ensure the optimization of adjustable parameters. For example, the output power was raised from a few watts of multi-mode to several thousand watts of near-diffraction limit in tens of years for flowing HF chemical lasers ${ }^{[5]}$. There are also great disparities in the chemical efficiencies of supersonic COIL experiments ${ }^{[11]}$. Herein spectral line broadening (SLB) model is one of the key factors in explaining the disparities besides the flowing factors and an appropriate SLB model can play an important role in optimizing the adjustable parameters. Thus, it is important to examine and develop different SLB models in the COIL.

About the chemical reaction system, Perram et al. ${ }^{[12]}$ presented a "standard" reaction system for COIL. There are as many as 53 reactions in it. Perram also presented a simplified reaction system with 13 reactions and compared it with the one with 53 reactions ${ }^{[13]}$, and concluded that the simplified reaction system can basically reflect the variation of density of all components in typical operating conditions. Carrol et al. ${ }^{[14]}$ and Paschkewitz et al. ${ }^{[15]}$ examined the effect of different reaction systems on the performance of COIL and believe that the reaction system with 8 reactions give a prediction of power on the high side. There are many means in choosing the reaction systems, and here a chemical reaction system of 10 components and 21 reactions is ultilized.

\section{Flow and chemical reaction model in flowing COIL}

In the COIL, the excited oxygen is produced by reaction of $\mathrm{KOH}$ (or $\mathrm{NaOH}$ ), chlorine and water vapour, and there is a mass of water vapour in the excited oxygen gas flow. The water vapour is a strong deactivator, and a mass of heat is produced in these deactivating reactions and this may lead to serious problems, i.e., "heat jam". Thus it is necessary to remove most water vapour with a condensator. After removing the water by 
a condensator, the excited oxygen is mixed with iodine vapour and a great mass of diluent, usually Heidner, or nitrogen in the reaction cavity, which are injected into the reaction cavity through rows of nozzles. There the mixing process and chemical reactions happen simultaneously. The design of nozzles is optimized and all the mediums can mix well in the distance before entering into the optical cavity; thus the gas flow can be simplified as premixed one dimensional flow. The temperature, gas density and flow velocity are assumed to be constant here.

The reactions in the reaction cavity are concerned with the solving reactions of iodine molecules and reactions of three oxygen energy levels with the two energy levels of iodine atoms, and also with other molecules. The whole reaction system is very complicated. A detailed study about chemical reaction system is presented in ref. [7], a reaction model used in ref. [16] including 21 reactions is used in this calculating model, and some of the rate constants also make use of refs. [13-15] as a reference. Table 1 presents the chemical kinetic and the reaction rate constants, including 10 components: $\mathrm{O}_{2}\left({ }^{1} \Sigma\right), \mathrm{O}_{2}\left({ }^{1} \Delta\right), \mathrm{O}_{2}\left({ }^{3} \Sigma\right), \mathrm{I}_{1} \mathrm{I}^{*}, \mathrm{H}_{2} \mathrm{O}, \mathrm{He}, \mathrm{Cl}_{2}, \mathrm{I}_{2}, \mathrm{I}_{2}{ }^{*}$. In the case where the iodine molecule is decomposed completely, the chemical reaction system is simplified to 8 components and 13 reactions $(1-12$ and 21$)$.

Table 1 Chemical reaction system and rate constants

\begin{tabular}{cll}
\hline Reaction & \multicolumn{1}{c}{ Reaction equations } & Rate constants (cc/s-molec) \\
\hline 1 & $\mathrm{I}^{+}+\mathrm{O}_{2}\left({ }^{1} \Delta\right) \rightarrow \mathrm{I}^{*}+\mathrm{O}_{2}\left({ }^{3} \Sigma\right)$ & $2.33 \times 10^{-8} / \mathrm{T}$ \\
2 & $\mathrm{I}^{*}+\mathrm{O}_{2}\left({ }^{3} \Sigma\right) \rightarrow \mathrm{I}+\mathrm{O}_{2}\left({ }^{1} \Delta\right)$ & $3.11 \times 10^{-8} / \mathrm{T} * \mathrm{exp}(-401.4 / \mathrm{T})$ \\
3 & $\mathrm{O}_{2}\left({ }^{1} \Delta\right)+\mathrm{O}_{2}\left({ }^{1} \Delta\right) \rightarrow \mathrm{O}_{2}\left({ }^{3} \Sigma\right)+\mathrm{O}_{2}\left({ }^{1} \Sigma\right)$ & $2.7 \times 10^{-17}$ \\
4 & $\mathrm{O}_{2}\left({ }^{1} \Sigma\right)+\mathrm{H}_{2} \mathrm{O} \rightarrow \mathrm{O}_{2}\left({ }^{1} \Delta\right)+\mathrm{H}_{2} \mathrm{O}$ & $6.7 \times 10^{-12}$ \\
5 & $\mathrm{O}_{2}\left({ }^{1} \Delta\right)+\mathrm{H}_{2} \mathrm{O} \rightarrow \mathrm{H}_{2} \mathrm{O}+\mathrm{O}_{2}\left({ }^{3} \Sigma\right)$ & $4 \times 10^{-18}$ \\
6 & $\mathrm{O}_{2}\left({ }^{1} \Delta\right)+\mathrm{O}_{2}\left({ }^{3} \Sigma\right) \rightarrow \mathrm{O}_{2}\left({ }^{3} \Sigma\right)+\mathrm{O}_{2}\left({ }^{3} \Sigma\right)$ & $1.6 \times 10^{-18}$ \\
7 & $\mathrm{O}_{2}\left({ }^{1} \Delta\right)+\mathrm{He} \rightarrow \mathrm{O}_{2}\left({ }^{3} \Sigma\right)+\mathrm{He}$ & $8 \times 10^{-21}$ \\
8 & $\mathrm{I}^{3}+\mathrm{O}_{2}\left({ }^{1} \Delta\right) \rightarrow \mathrm{I}+\mathrm{O}_{2}\left({ }^{3} \Sigma\right)$ & $5 \times 10^{-14}$ \\
9 & $\mathrm{I}^{*}+\mathrm{O}_{2}\left({ }^{1} \Delta\right) \rightarrow \mathrm{I}+\mathrm{O}_{2}\left({ }^{1} \Delta\right)$ & $1.1 \times 10^{-13}$ \\
10 & $\mathrm{I}^{*}+\mathrm{O}_{2}\left({ }^{1} \Delta\right) \rightarrow \mathrm{I}+\mathrm{O}_{2}\left({ }^{1} \Sigma\right)$ & $1.1 \times 10^{-13}$ \\
11 & $\mathrm{I}^{+}+\mathrm{I}^{*} \rightarrow \mathrm{I}+\mathrm{I}$ & $1.6 \times 10^{-14}$ \\
12 & $\mathrm{I}^{*}+\mathrm{H}_{2} \mathrm{O} \rightarrow \mathrm{I}+\mathrm{H}_{2} \mathrm{O}$ & $2 \times 10^{-12}$ \\
13 & $\mathrm{I}_{2}+\mathrm{O}_{2}\left({ }^{1} \Sigma\right) \rightarrow 2 \mathrm{I}+\mathrm{O}_{2}\left({ }^{3} \Sigma\right)$ & $3.9 \times 10^{-11}$ \\
14 & $\mathrm{I}_{2}{ }^{*}+\mathrm{O}_{2}\left({ }^{1} \Delta\right) \rightarrow 2 \mathrm{I}+\mathrm{O}_{2}\left({ }^{3} \Sigma\right)$ & $3 \times 10^{-10}$ \\
15 & $\mathrm{I}_{2}+\mathrm{O}_{2}\left({ }^{1} \Sigma\right) \rightarrow \mathrm{I}_{2}+\mathrm{O}_{2}\left({ }^{1} \Delta\right)$ & $1.6 \times 10^{-11}$ \\
16 & $\mathrm{I}_{2}+\mathrm{O}_{2}\left({ }^{1} \Delta\right) \rightarrow \mathrm{I}_{2}{ }^{*}+\mathrm{O}_{2}\left({ }^{3} \Sigma\right)$ & $7 \times 10^{-15}$ \\
17 & $\mathrm{I}_{2}{ }^{*}+\mathrm{O}_{2}\left({ }^{3} \Sigma\right) \rightarrow \mathrm{I}_{2}+\mathrm{O}_{2}\left({ }^{3} \Sigma\right)$ & $4.9 \times 10^{-12}$ \\
18 & $\mathrm{I}_{2}{ }^{*}+\mathrm{H}_{2} \mathrm{O} \rightarrow \mathrm{I}_{2}+\mathrm{H}_{2} \mathrm{O}$ & $7 \times 10^{-11}$ \\
19 & $\mathrm{I}_{2}{ }^{*}+\mathrm{He}^{3} \rightarrow \mathrm{I}_{2}+\mathrm{He}^{*}$ & $9.8 \times 10^{-12}$ \\
20 & $\mathrm{I}_{2}+\mathrm{I}^{*} \rightarrow \mathrm{I}+\mathrm{I}_{2}{ }^{*}$ & $3.8 \times 10^{-11}$ \\
21 & $\mathrm{O}_{2}\left({ }^{1} \Delta\right)+C \mathrm{Cl}_{2} \rightarrow \mathrm{O}_{2}\left({ }^{3} \Sigma\right)+\mathrm{Cl}{ }_{2}$ & $6 \times 10^{-18}$ \\
Photon transition & $\mathrm{I}^{*}+\mathrm{hv}^{3} \rightarrow \mathrm{I}+2 \mathrm{hv}$ & $5 \mathrm{~s}{ }^{-1}$ \\
\hline
\end{tabular}


In table 1, those reactions of components $X_{i}$ which do not participate in laser radiation can be expressed with a common equation:

$$
\sum_{i=1}^{n j} \alpha_{r i} X_{i} \Rightarrow \sum_{i=1}^{n j} \beta_{r i} X_{i}
$$

Here all reactions are dealt with as irreversible and the reversible pumping reaction is regarded as 2 reactions. The producing rate of the density of the $i$-th component (denoted with [.]) which does not participate in laser radiation can be expressed as

$$
u \frac{\partial\left[X_{i}\right]}{\partial x}=\sum_{r=1}^{n r}\left(\beta_{r i}-\alpha_{r i}\right) k_{r i} \prod_{j=1}^{n j}\left(\left[X_{j}\right]\right)^{\alpha_{r j}} .
$$

Here the suffix $r$ is the serial number of chemical reactions, $\alpha_{r i}$ and $\beta_{r i}$ are the molar ratio of the $i$-th component of reactants and resultants, $k_{r}$ is the rate constants of the $i$-th reaction, $u$ is the gas flow velocity and $x$ is the coordinate streamwise direction. $h$ and $v$ are the Plank constant and frequency of photons.

The equilibrium constant of reactions (1) and (2) $K_{e}=0.75 \exp (401.4 / T)$ (here $T$ (unit: $K$ ) is the gas temperature), $K_{e}=2.9$ at room temperature $(298 \mathrm{~K})$, and in this case a population inversion exists in chemical equilibrium if the content of $\mathrm{O}_{2}\left({ }^{1} \Delta\right)$ is greater than $15 \%$ of the total oxygen. Generally, the temperature in a flowing COIL cavity is lower than the room temperature and it is favorable to the population inversion. The concentration of atomic iodine is about $3 \%$ of that of molecular oxygen and each iodine atom is repumped and cycled many times throughout the flow field during the lasing process.

If the iodine molecule is assumed to be decomposed completely and the iodine atom is a two energy level system, then

$$
n_{1}+n_{2}=n_{0},
$$

where $n_{1}$ and $n_{2}$ are the population of the ground and excited state (or the lower and upper energy level) of the atomic iodine, respectively. $n_{0}$ is the total population of atomic iodine. The total population of atomic iodine, $n_{0}$, is constant if all the iodine molecule is decomposed, and increased as the iodine molecule is decomposed when iodine molecules existed at the entrance.

\section{Gain saturation equation for supersonic COIL performance modeling-deduc- tion of SGK model}

In the SGK model, the gas kinetic equations that describe the translational velocity distributions of particles in the upper and lower iodine energy levels are presented and a method for solving these equations is developed. In the deduction of this model, a gain coefficient related to the translational velocity of lasing particles is introduced: 


$$
g_{T}=\sigma \phi\left(v, v_{0}\right)\left(f_{2}-\alpha f_{1}\right)
$$

where $\sigma$ is the stimulated emission cross section which is dependent on the pressure, $f_{i}(i$ $=1,2)$ is the velocity distribution of upper and lower laser level, respectively. $\phi\left(v, v_{0}\right)$ is the Lorentz profile function, $\alpha$ is a constant related to level degeneracy, for the COIL $\alpha \approx 1 / 2$, and $v$ and $v_{0}$ are the laser radiation frequency and the central frequency of the spectral line profile, respectively. According to eq. (1), it can be assumed reasonably

$$
f_{1}+f_{2}=f,
$$

where $f$ is the total velocity distribution function of particles in the upper and lower laser energy levels. From eqs. (3) and (4), we have

$$
\begin{aligned}
& f_{1}=\frac{1}{1+\alpha}\left(f-\frac{g_{T}}{\sigma \phi}\right), \\
& f_{2}=\frac{1}{1+\alpha}\left(\alpha f+\frac{g_{T}}{\sigma \phi}\right) .
\end{aligned}
$$

The kinetic equations for the electronic energy levels and the equation of radiative transfer can be written respectively as

$$
\begin{gathered}
u \frac{\partial f_{2}}{\partial x}=r f_{1}+k_{e}\left(f_{2}^{0}-f_{2}\right)-k_{p} f_{2}-\sigma \phi\left(v, v_{0}\right)\left(f_{2}-\alpha f_{1}\right) \frac{I}{h v} \\
u \frac{\partial f_{1}}{\partial x}=-r f_{1}+k_{\mathrm{e}}\left(f_{1}^{0}-f_{1}\right)+k_{\mathrm{p}} f_{2}+\sigma \phi\left(v, v_{0}\right)\left(f_{2}-\alpha f_{1}\right) \frac{I}{h v}+k_{\mathrm{s}}, \\
c \frac{\partial f_{v}}{\partial x}=f_{v} \int_{-\infty}^{+\infty} \sigma\left(f_{2}-\alpha f_{1}\right) d v_{T y},
\end{gathered}
$$

where $f_{i}^{0}(i=1,2)$ is the equilibrium distribution function (i.e. Maxwellian distribution function). $k_{e}$ is the elastic collision characteristic rate and it is proportional to the number density of all particles and the collision-frequency. $k_{p}$ is the deactivation rate, $r$ is the pumping rate of the upper laser level. $I$ is the optical intensity. $c$ and $v_{T y}$ are velocity of light and thermal translation velocity of laser particles along the centerline of the resonator mirrors, respectively. $f_{v}$ is the frequency distribution function of photons and $k_{s}$ is the increment rate of iodine atom of per unit interval of translational velocity in per unit volume obtained from the decomposition of iodine molecules. Here $r=k_{f} n_{\Delta}$,

$$
k_{p}=k_{2}\left[O_{2}\left({ }^{3} \Sigma\right)\right]+k_{9}\left[O_{2}\left({ }^{1} \Delta\right)\right]+k_{10}\left[O_{2}\left({ }^{1} \Delta\right)\right]+k_{11} n_{1}+k_{12}\left[H_{2} O\right]+k_{20}\left[I_{2}\right] .
$$

The equation for $g_{T}$ can be deduced from eq. (6) as 


$$
u \frac{\partial g_{T}}{\partial x}+\left[\left(r+k_{p}\right)+(1+\alpha) \frac{\sigma \phi I}{h v}\right] g_{T}=\left(r-\alpha k_{p}\right) \sigma \phi f
$$

It is impossible to obtain an analytical solution from eq. (8). A double-parameter perturbation method is used to solve eq. (8) and the velocity distribution function $f_{i}(i=1$, 2) can be expanded to power-series of double parameters $u / L K_{\nu}$ and $u / L K_{e}$

$$
f_{i}=\sum_{j=0}^{\infty} \sum_{k=0}^{\infty}\left(\frac{u}{L k_{v}}\right)^{j}\left(\frac{u}{L k_{e}}\right)^{k} f_{i}^{j k} .
$$

Here $k_{v}$ is the radiation characteristic rate. $L$ is the length of the resonator in the streamwise direction. The half-order relations can be obtained from eqs. (6) and (8) as

$$
\begin{gathered}
f_{2}^{0}-\alpha f_{1}^{0}=\phi\left(f_{2}^{00}-\alpha f_{1}^{00}\right)\left(1+\frac{2 \sigma}{h v k_{e}} \phi I\right)^{-1}, \\
f_{2}^{00}-\alpha f_{2}^{0}=\frac{\sigma I}{h v k_{e}} \phi\left(f_{2}^{00}-\alpha f_{1}^{00}\right), \\
f_{1}^{00}-\alpha f_{1}^{0}=\frac{\sigma I}{h v k_{e}} \phi\left(f_{2}^{00}-\alpha f_{1}^{00}\right), \\
g_{T}^{0}=\sigma \phi\left(v^{\prime}, v\right)\left(f_{2}^{00}-\alpha f_{1}^{00}\right) .
\end{gathered}
$$

Because the gain

$$
g=\int_{-\infty}^{+\infty} g_{T} d v_{T y}
$$

is equal to the loss in the laser cavity for a Fabry-Perot resonator, and the loss is independent of the position (the constant gain approximation assumption) ${ }^{[1,5,6]}$, there are

$$
g=g_{\text {th }}, \frac{\partial g}{\partial x} \approx 0
$$

From eqs. (7), (11) and (9), integrating the gain with respect to the apparent frequency, a gain saturation equation is obtained as

$$
g=K \sigma n_{0} \frac{\eta^{2}(1+\bar{I})}{\sqrt{\pi}} \int_{-\infty}^{+\infty} \frac{e^{-t^{2}}}{\eta^{2}(1+\bar{I})+(\xi-t)^{2}} d t,
$$

where $K=\left(r-\alpha k_{p}\right) /\left(r+k_{p}\right) ; \quad \bar{I}$ is the dimensionless saturation optical intensity. $\bar{I}=I / \tilde{I}, \quad \tilde{I}$ is the saturation optical intensity and $\tilde{I}=2 h v\left(k_{f} n_{\Delta}+k_{r} n_{\Sigma}\right) / 3 \sigma ; \quad \eta$ is the broadening parameter and it is equal to $\sqrt{\ln 2} \Delta v_{N} / \Delta v_{D}$; frequency-shift parameter 
$\xi=2 \sqrt{\ln 2}\left(v-v_{0}\right) / \Delta v_{D}, \quad t=2 \sqrt{\ln 2}\left(v^{\prime}-v_{0}\right) / \Delta v_{D}$ is a temporal integral variable, $\Delta v_{N}$ and $\Delta v_{D}$ are the whole width at half height of the Lorentz and Doppler broadening spectral line profile, respectively.

In the case of laser radiation frequency coinciding with the central frequency of the line profile, i.e. the frequency-shift parameter $\xi=0$, it can be deduced from eq. (12) that

$$
g=K \sigma n_{0} \frac{\eta \sqrt{\pi}}{\sqrt{1+\bar{I}}} \exp \left[(1+\bar{I}) \eta^{2}\right] \operatorname{erfc}(\eta \sqrt{1+\bar{I}})
$$

When $\eta>>1$, i.e. the homogeneous broadening being predominant (high pressure limit), expanding the error function in eq. (13) gives

$$
g=\frac{K \sigma n_{0}}{1+\bar{I}}
$$

When $\eta<<1$, i.e. the inhomogeneous broadening being predominant (low pressure limit), eq. (13) gives

$$
g=K \sigma n_{0} \frac{\eta \sqrt{\pi}}{\sqrt{1+\bar{I}}}
$$

These approximate expressions at high and low gas pressure are consistent with the gain saturation expressions of the gas laser ${ }^{[17]}$.

Under the approximation that the near-resonant energy transition reaction is always in a balanced status, the rate of variation of excited oxygen density along streamwise distance is

$$
u \frac{d Y}{d x}=-\frac{g I}{h v\left[O_{2}\right]}-k_{c}
$$

where $Y$ is the yield of excited oxygen, $Y=n_{\Delta} /\left[O_{2}\right]$ and $n_{\Delta}$ is the density of excited oxygen, $k_{c}$ is the rate of consuming $\mathrm{O}_{2}\left({ }^{1} \Delta\right)$ by chemical reactions,

$$
\begin{aligned}
k_{c}= & \left(2 k_{3}\left[O_{2}\left({ }^{1} \Delta\right)\right]+k_{5}\left[H_{2} O\right]+k_{6}\left[O_{2}\left({ }^{3} \Sigma\right)\right]+k_{7}\left[H_{e}\right]+k_{8} n_{1}+k_{10} n_{2}+k_{14}\left[I_{2}^{*}\right]+k_{16}\left[I_{2}\right]\right. \\
& \left.+k_{21}\left[C l_{2}\right]\right)\left[O_{2}\left({ }^{1} \Delta\right)\right]+\left(2 k_{9}\left[O_{2}\left({ }^{1} \Delta\right)\right]+k_{10}\left[O_{2}\left({ }^{1} \Delta\right)\right]+k_{11}[I]+k_{12}\left[H_{2} O\right]\right. \\
& \left.+k_{20}\left[I_{2}\right]\right)\left[I^{*}\right]-\left(k_{15}\left[I_{2}\right]+k_{4}\left[H_{2} O\right]\right)\left[O_{2}\left({ }^{1} \Sigma\right)\right] .
\end{aligned}
$$

By combining eq. (14), (17) and the boundary condition of oxygen, the variation of excited oxygen yield $Y$, light intensity $I$ along flow direction can be obtained. The calculation of the power can use ${ }^{[10]}$

$$
P=\eta_{\text {extr }} L H \int_{0}^{l_{r}} g I d x
$$




\section{Comparison with the RE model}

According to ref. [5], the gain relationship of the RE model can be deduced as

$$
g=K \sigma n_{0} \frac{\eta \sqrt{\pi} \operatorname{erfc} \eta \exp \left(\eta^{2}\right)}{1+\bar{I} \eta \sqrt{\pi} \operatorname{erfc} \eta \exp \left(\eta^{2}\right)} .
$$

When $\eta>>1$, i.e. the homogeneous broadening being predominant (high pressure limit), expanding the error function in eq. (19), an equation that is the same as eq. (15) can be obtained. When $\eta<<1$, i.e. the inhomogeneous broadening being predominant (low pressure limit), eq. (19) gives approximately

$$
g=\frac{\eta \sqrt{\pi}\left(K \sigma n_{0}\right)}{1+\bar{I} \eta \sqrt{\pi}} .
$$

It is drastically different from that of the SGK model under the condition of low gas pressure.

\section{Results and discussions}

For the performance modeling of COIL, we use the experimental and calculating data of RotoCOIL ${ }^{[3,16]}$. The extraction efficiency of the resonator is

$$
\eta_{\text {extr }}=\left(1-R_{\text {out }}-S_{\text {out }}\right) /\left[\left(1-R_{\text {out }}-S_{\text {out }}\right)(1+\delta)+S_{\text {out }}+\left(R_{\text {out }} / R_{\max }\right)^{1 / 2}\left(1-R_{\max }\right)\right] .
$$

In this experiment, the reflectivity of the highly reflective mirror $R_{\max }=0.995$, the scattering coefficient of the outcoupling mirror $S_{\text {out }}=0.004$, and the diffraction loss fraction $\delta=0.13^{[3]}$. The flow conditions within the laser cavity are deduced from calculation of the RotoCOIL experimental data ${ }^{[3]}$ : the gas temperature $T$ is $167 \mathrm{~K}$, the gas pressure $P$ is approximately 4 torrs, the small signal gain at the entrance is $0.0068 \mathrm{~cm}^{-1}$, the yield $Y_{0}$ $=0.42$, the molar flow rate of oxygen $Q=1.395 \mathrm{~mol} / \mathrm{sec}$, the dilution ratio is approximately He: $\mathrm{O}_{2}=4: 1$, the flow ratio of $\mathrm{I}_{2}$ and $\mathrm{O}_{2}$ is $\mathrm{I}_{2} / \mathrm{O}_{2}=0.0158$ and flow velocity $u=$ $841 \mathrm{~m} / \mathrm{s}^{[14]}$. According to a numerical simulation ${ }^{[14]}$ of the experiment, the broadening parameter $\eta$ is about 0.08 according to estimation of calculation. The calculation herein is based on the RotoCOIL experiment, that is, choosing parameters whose effects are considered and their related variables are determined on the basis of experimental data and other parameters are directly taken as the experimental data.

Fig. 1 shows a comparison of the RotoCOIL experimental data with the calculation results of four theoretical models. The four models are RE and SGK models considering 21 reactions in this paper, the SGK and low pressure limit of RE model in which only the reversible pumping reactions are considered (denoted with SGK1 and low pressure limit model in this paper for advantage, respectively). The calculating conditions are the same as experimental data. It shows that in the whole range of threshold gain where power extraction exists, the output power values of all theoretical models are consistent 
with the experimental data approximately, but the results of the SGK and SGK1 models are better than those of the RE and low pressure limit of RE models, the optimal threshold gain range (the power $P$ satisfies $\left|P-P_{\max }\right| \cong 0.1, P_{\max }$ is the maximum output power) of low pressure limit model, RE, and SGK and SGK-model are: $0.00044-0.00250$, $0.00043-0.00235,0.00039-0.001760 .00041-0.00185\left(\mathrm{~cm}^{-1}\right)$, respectively. These data indicate that there is comparatively great difference of the predicted optimal threshold gain range between different models, especially the RE and SGK models. Because the threshold gain decides the value of output power, herein to predict the optimal threshold gain exactly is very important to the extraction of power and the design of the optical cavity. It also indicates that the inhomogeneous broadening effect of low pressure has great effect on the performance of COIL.

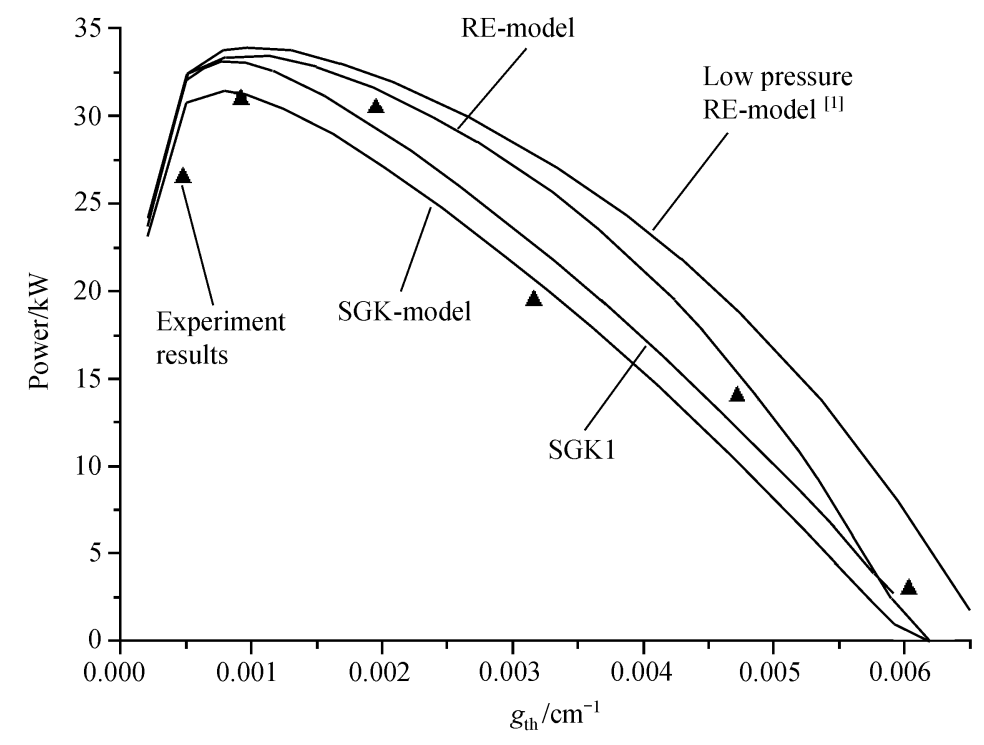

Fig. 1. Comparison of papers predicted by four models with RotoCOIL experimental data.

Fig. 2 shows the effect of frequency-shift $\xi$ on the output power. The existence of frequency-shift decreases the output power, and reduces the threshold gain range that output power exists in all the models. RE model's results are obviously greater than that of SGK model, and SGK1 model's results are lower than that of RE model but slightly higher than that of SGK model.

Fig. 3 shows the effect of the water concentration on the output power. In the case of high water concentration, the existence of water decreases the output power of COIL obviously. The output powers of both RE and SGK model decrease as water concentration increases, but results of SGK model are obviously lower than that of RE model.

Fig. 4 shows variations of output power with threshold gain when some un- 


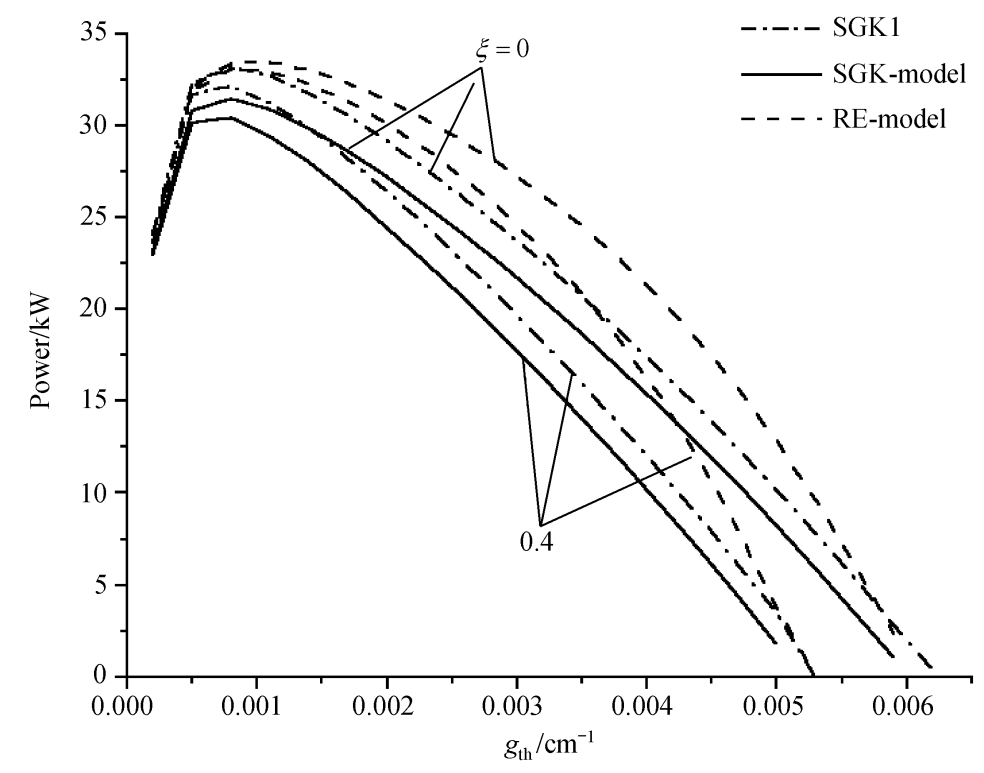

Fig. 2. Variations of powers with $g_{\mathrm{th}}$ for different frequency shift $\xi$.

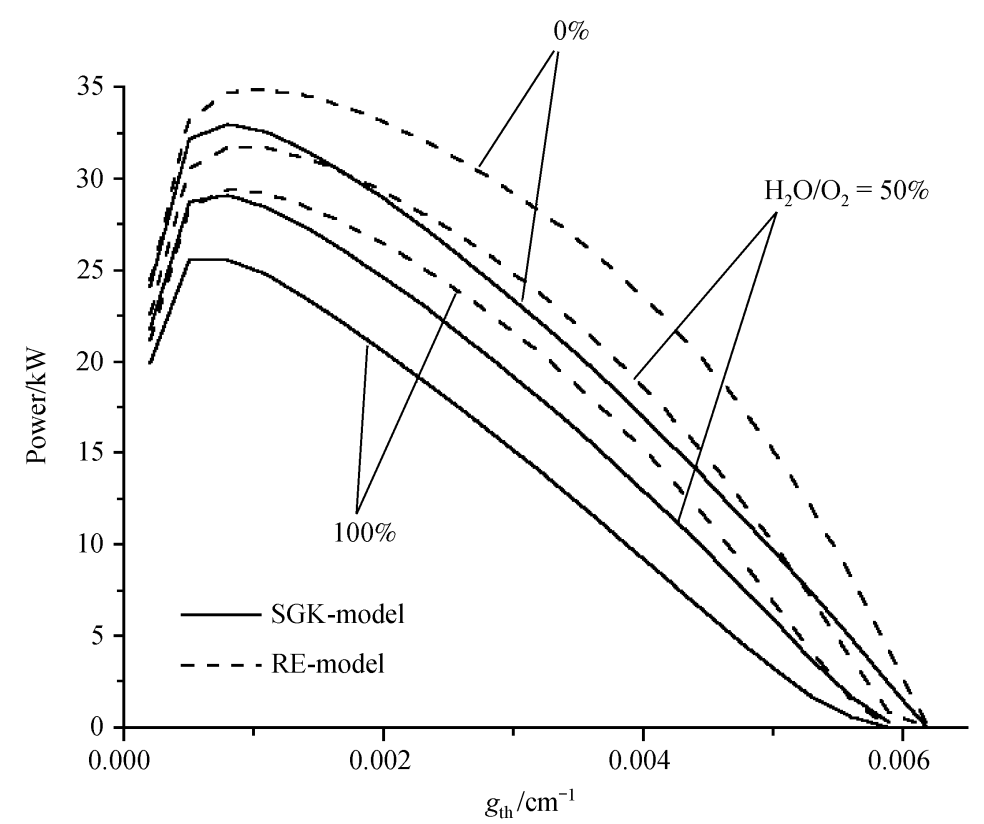

Fig. 3. Variations of SGK- and RE-models'power with $g_{\text {th }}$ for different content of water.

decomposed iodine molecule exists at the entrance of the optical cavity. The curves of this figure indicate that the existence of un-decomposed iodine molecule is useful to the extraction of power. The output power increases as increment of concentration of 
un-decomposed iodine molecules. This is because the decomposition of iodine molecules increases the concentration of iodine atoms and herein increases the gain value, which is favorable to the extraction of power. But the output power will decrease if the concentration of un-decomposed iodine molecules is too high, and the output power will decrease in low threshold gain case.

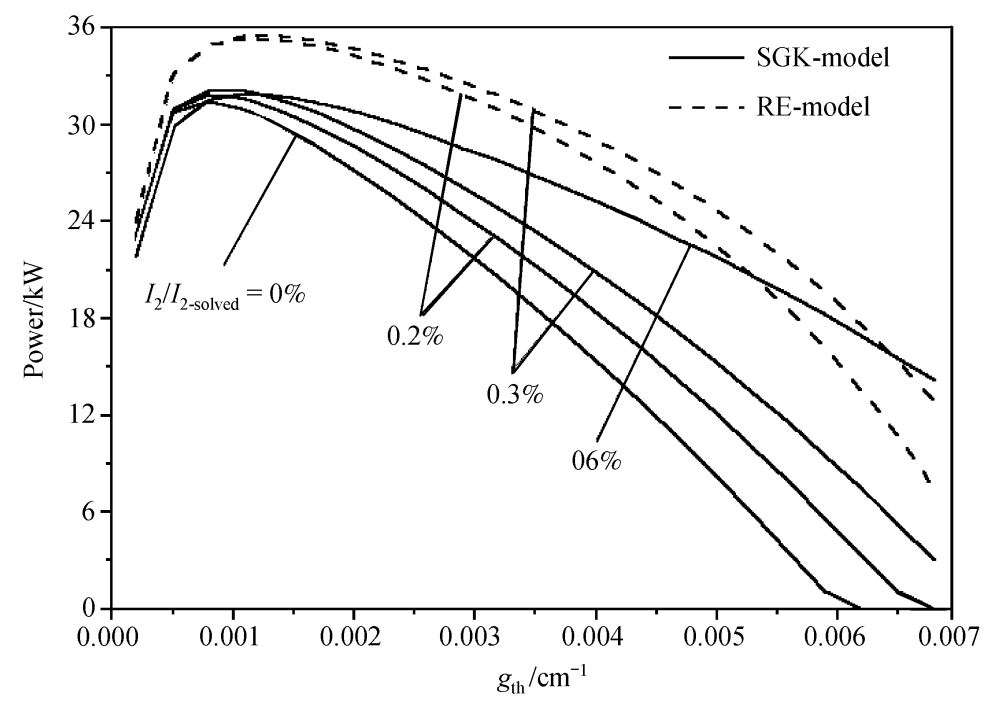

Fig. 4. Variations of SGK- and RE-model's power with $g_{\text {th }}$ for different content of un-decomposed iodine molecule.

Fig. 5 shows variation of the output power with $g_{\text {th }}$ for different yield of the excited oxygen $\mathrm{O}_{2}\left({ }^{1} \Delta\right)$ at the entrance of the cavity, i.e. $Y_{0}$. It is shown that as $Y_{0}$ increases, the threshold gain at zero power crossing point increases slightly, but the output power increases obviously in both the SGK model and the RE models. Chemical reactions also affect the output power for SGK model's results are lower than that of SGK1.

Fig. 6 shows variations of the output power with the threshold gain $g_{\text {th }}$ for different temperature $T$. As the temperature decreases, the output power increases in all RE, SGK and SGK1 models. As temperature decreases, the threshold gain range with high output power also increases obviously. Therefore, a decrease in temperature by means of increasing the expansion Mach number is important for improving performances of the COIL.

Fig. 7 shows respectively variations of the output power, the optical intensity $I$, the saturation degree $S$ (it is defined as $1-g_{\text {th }} / g_{00}$ when strong light signal exists ${ }^{[17]}$ ) and the $\mathrm{O}_{2}\left({ }^{1} \Delta\right)$ yield $Y$ with the distance downstream by using the RE, SGK and the SGK1 models. Here $g_{\text {th }}=0.002 \mathrm{~cm}^{-1}$ and other values are taken as experimental data.

Fig. 7(a) shows that in all models, the output power increase accelerates near the entrance, and slows down downstream. There is hardly any difference between the out- 


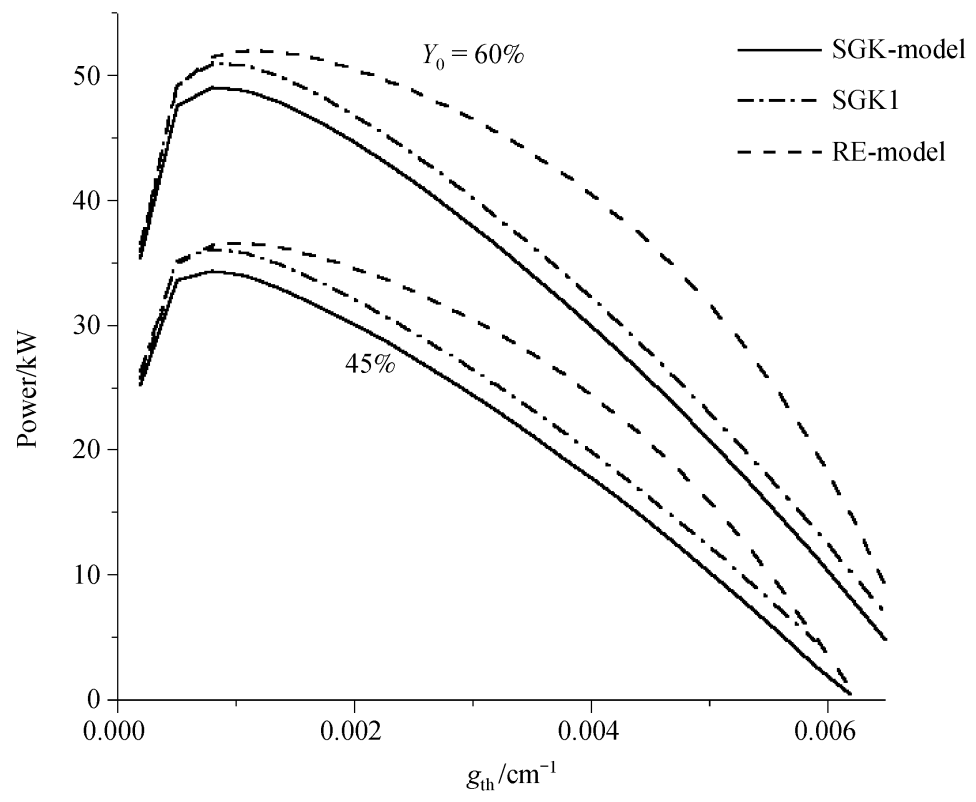

Fig. 5. Variations of power with $g_{\text {th }}$ for different yield.

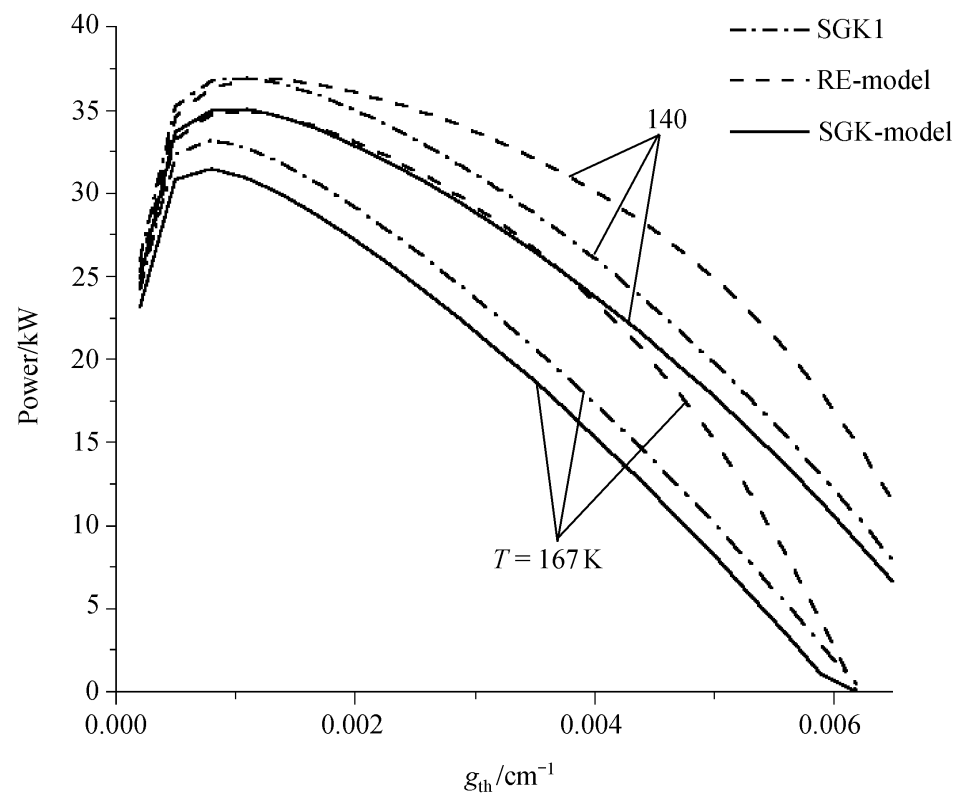

Fig. 6. Variations of power with $g_{\text {th }}$ for different temperature.

put power of SGK and SGK1 model upstream, and output power of SGK model is slightly lower downstream. The output power of the SKG and SGK1 models increases all along the mirror length but it is obvious that the power extraction of the RE model is much quicker and is nearly completed in half of the mirror length. The great difference 

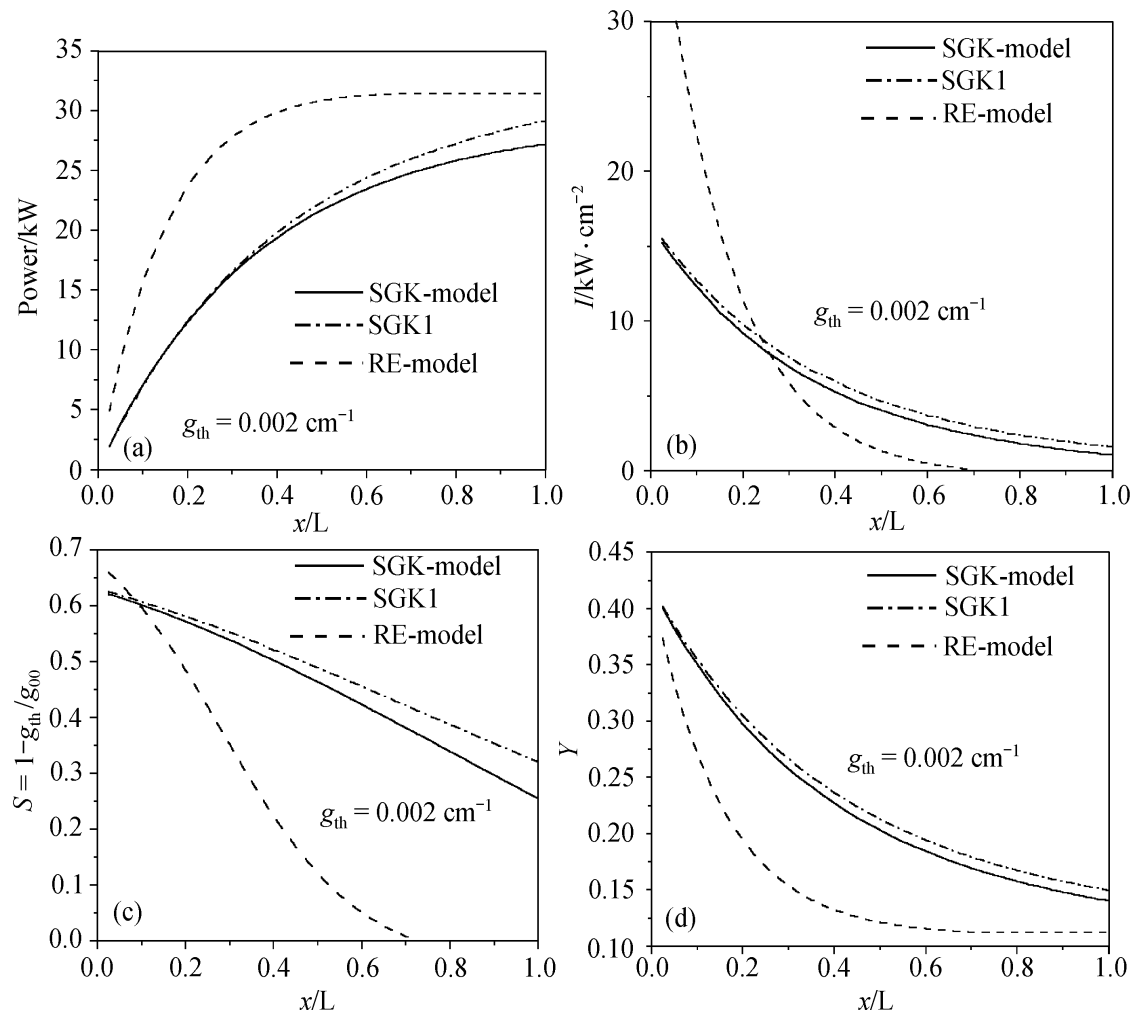

Fig. 7. Variations of extraction power increment (a), flux intensity (b), saturation parameter (c) and excited oxygen yield (d), with the streamwise $x$ - direction with $g_{\text {th }}=0.002$ ( $L$ is the length of the resonator).

of power extraction length between SGK and RE model indicates the importance of gain saturation model in the design of optical cavity. Fig. 7(b) shows that the optical intensity of the RE model is much greater than that of the other two models near the entrance and smaller at the exit, and it decreases more rapidly along the streamwise direction. Results of SGK and SGK1-model are approximately the same.

Fig. 7(c) shows the SGK and SGK1 model's saturation degree is greater than that of the RE model, and they decrease more slowly, too. As to the variations of the oxygen yield in fig. 7(d), the SGK model predicts a slower decrease and more residual energy of excited oxygen remains in the exhaust gas that flows out of the laser cavity. There is little difference in the yield of excited oxygen between SGK and SGK1 models in all positions.

Acknowledgements This research is supported by the National Natural Science Foundation of China (Grant No. 10032050).

\section{References}

1. Hager, G. D., Helms, C. A., Truesdell, K. A., et al., A simplified analytic model for gain saturation and power extraction in the flowing chemical oxygen-iodine laser, IEEE Journal of Quantum Electronics, 1996, 32(9): 
1525-1536.[DOI]

2. Barmashenko, B. D., Rosenwaks, S., Analysis of the optical extraction efficiency in gas-flow lasers with different types of resonators, Applied Optics, 1996, 35(36): 7091-7101.

3. Zhuang, Q., Sang, F. T., Zhou, D. Z., Short-Wavelength Chemical Lasers (in Chinese), Beijing: National Defence Industry Press, 1997.

4. Li, S. X., Zhang, B. A., The effects of iodine density on COIL central transition frequencies, High Power Laser and Particle Beams, 1999, 11(3): 262-265.

5. Gross, R. W. F., Bott, J. F., Handbook of Chemical Lasers, New York: Willey, 1976, 501.

6. Gao Zhi, Collisional and inhomogeneous broadening effects in gas-flow and chemical lasers: The theoretical models, Acta Physica Sinica (in Chinese), 1981, 30(12): 1591-1602.

7. Gao, Z., E, X. Q., Kinetic model of continuous-wave flow chemical lasers, Science in China (Series A), 1982, 25(2): $171-185$.

8. E, X. Q., Gao, Z., Effects of inhomogeneous broadening of the performance of CW chemical lasers, Acta Physica Sinica, 1983, 3(6): 513-520.

9. Fan, A. F., Gao, Z., Gain saturation effects in nonequilibrium gas flow and chemical lasers, Acta Physica Sinica (Series A), 1993, 42(3): 407-416. (in Chinese)

10. Fan, A. F., Gao, Z., Zhou, X., Cross-relaxation dynamics on anomalous saturation processes in low pressure supersonic cw HF chemical laser magnifier, Science in China (Series A), 1998, 41(10): 1083-1091.

11. Hon, J. F., Plummer, D. N., Crowell, P. G., A heuristic method for evaluation COIL performance, AIAA-25th Plasmadynamics and Laser Conf. Colorado Springs. CO. June 20-23, Washington: American Institute of Aeronautics and Astronautics, 1994, paper 94-2422.

12. Perram, G. P., Hager, G. D., The standard chemical oxygen-iodine laser kinetics package, USAF Technical Report AFWL-TR-88-50, 1988.

13. Perram, G. P., Approximate analytic solution for the presence of singlet oxygen, Int. J. Chem. Kinetics, 1995, 27(2): $817-828$.

14. Carrol, D., Modeling high-pressure chemical oxygen-iodine lases, AIAA Journal, 1995, 33(8): 1454-1462.

15. Paschkewitz, J., Shang, J., Miller, J. et al., An assessment of COIL physical property and chemical kinetic modeling methodologies, AIAA-31st Plasmadynamics and Laser Conf. Denver. CO. June 19-22, Washington: American Institute of Aeronautics and Astronautics, 2000, paper 2000-2574.

16. Buggeln, R. C., Shamroth, S., Lampson, A. et al., Three-dimensional(3-D) Navier-Stokes analysis of the mixing and power extraction in a supersonic chemical oxygen-iodine laser(COIl) with transverse $\mathrm{I}_{2}$ injection, AIAA 25th Plasmadynamics and Laser Conf., Colorado Springs, CO. June 20-23, Washington: American Institute of Aeronautics and Astronautics, 1994, paper 94-2435.

17. Chen, Y. Q., Wang, J. H., Principles of Lasers (in Chinese), Hangzhou: Zhejiang University Press, 1998, 299 -300 .

18. Yang, T. T., Copeland, D. A., Bauer, A. H. et al., Chemical oxygen-iodine laser performance modeling, AIAA Jour., 1996, 34(2): 338-346. 